\title{
Inhibition of Toxic Effects of Oxalate in Sugar Beet Pulp with Adding Ca During Ensiling
}

\author{
Cüneyt TEMÜR ${ }^{\star 1}$, Mehtap GÜNEY ${ }^{1}$, Sibel ERDOĞAN ${ }^{1}$, Murat DEMİREL ${ }^{1}$
}

${ }^{1}$ Yuzuncu Yil University Faculty of Agriculture Department of Animal Science, Van, TURKEY

Cüneyt TEMÜR, ORCID No:0000-0001-7952-7566, Mehtap GÜNEY, ORCID No: 0000-0002-0613-3600, Sibel ERDOĞAN, ORCID No: 0000-0003-2640-3871, Murat DEMİREL, ORCID No: 0000-0002-2992-8393

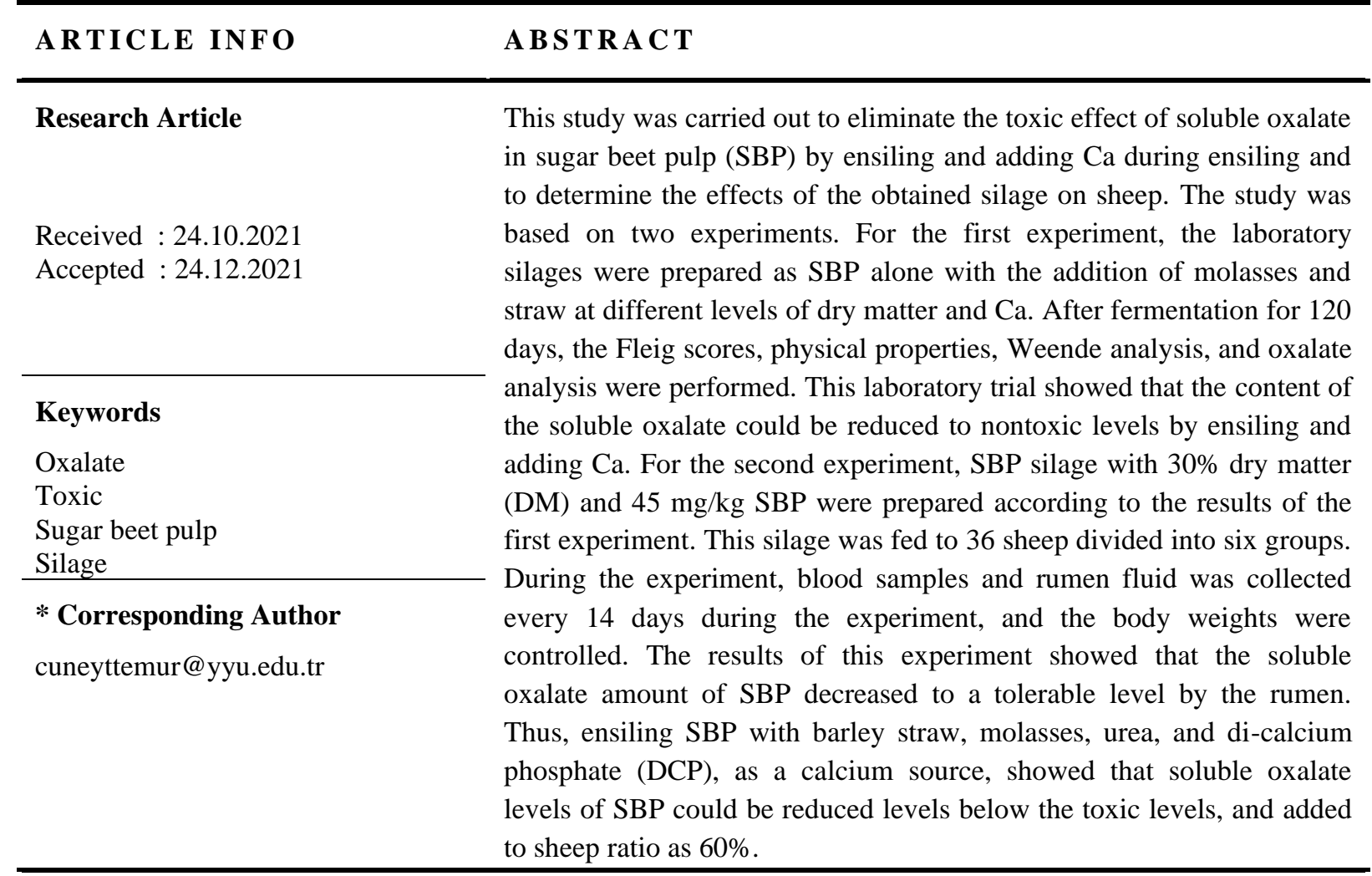

\section{Şeker Pancarı Posasında Bulunan Oksalatın Toksik Etkisinin Silolama ve Ca ilavesi ile Giderilmesi}
MAKA LE B İ L GIS İ
ÖZ

Araştırma Makalesi

$\mathrm{Bu}$ çalışma, şeker pancarı posasının (ŞPP) silolama ve silolama sırasında kalsiyum (Ca) ilavesinin çözünebilir oksalat üzerine etkilerini belirlemek ve bu silajın koyunlar üzerindeki etkilerini belirlemek amacıyla yapılmıştır. Çalışma iki deneme halinde yürütülmüştür. İlk deneme tek başına ŞPP silajı ile farklı kuru madde ve Ca seviyelerinde üre, melas ve saman ilavesi ile laboratuvar silajları olarak hazırlanmıştır.

Lütfen aşağıdaki şekilde atıf yapınız / Please cite this paper as following;

Temür, C., Güney, M., Erdoğan, S., Demirel, M., 2021. Inhibition of toxic effects of oxalate in sugar beet pulp with adding Ca during ensiling, Journal of Animal Science and Products (JASP) 4 (2):164-173. 


\begin{tabular}{ll}
\hline Anahtar Kelimeler & 120 günlük fermentasyondan sonra Fleig puanları, fiziksel özellikler, \\
Okzalat & Weende analizleri ve oksalat analizleri yapılmıştır. Bu laboratuvar \\
Toksik & denemesi, silolama ve Ca ilavesinin çözünür oksalat miktarının, \\
Şeker pancarı posası & ruminantlar için toksik olmayan seviyelere düşürülebileceğini \\
Silaj & göstermiştir. İkinci deneme için birinci denemenin sonuçlarına göre \%30 \\
& kuru madde (DM) ve $45 \mathrm{mg} / \mathrm{kg}$ ŞPP Ca içeren ŞPP silajı hazırlanmıştır. \\
\hline \multirow{2}{*}{ Sorumlu Yazar } & Bu silaj altı gruba ayrılan 36 koyuna yedirilmiştir. Deneme süresince 14 \\
cuneyttemur@yyu.edu.tr & günde bir kan örnekleri ve rumen sıvısı alınmış, vücut ağırlıkları kontrol \\
& edilmiştir. Yapılan iki denemenin sonuçları, ŞPP'nin çözünür oksalat \\
& miktarının rumen tarafından tolere edilebilir bir düzeye \\
& düşürülebileceğini göstermiştir. Bu nedenle, ŞPP’na arpa samanı, melas, \\
& üre ve kalsiyum kaynağ1 olarak dikalsiyum fosfat (DCP) ilavesi ile \\
& silajlanarak çözünür oksalat düzeylerinin toksik seviyelerin altına \\
& düşürülebileceği ve koyun rasyonlarına \%60 oranında katılabileceği \\
& kanaatine varılmıştır.
\end{tabular}

\section{Introduction}

Oxalates exist in the plant cell as metabolic end products such as soluble salts or insoluble salts. Oxalate accumulates as microscopic crystals in organs and tissues of many varieties of plants and comprise 85\% of DM among the species (Azcarate-Peril et al., 2006). The sensitivity of ruminants to plants with oxalates is variable. Sheep are more sensitive to oxalates than cattle (Mc Kenzie et al., 1988; Rahman et al., 2013). The amount of plant eaten, eating time, soluble oxalate content, the existence of other feed as a dilution factor in the rumen, and previous sensitivity to oxalate are the effective factors that determine the sensitivity of ruminants. Soluble oxalate on an empty stomach as $1 \mathrm{~g} / \mathrm{kg}$ may be sufficient for oxalate toxicity in animals (Kaya and Pirinçci, 1995; Rahman et al., 2013). Although there is no poisoning in animals, soluble oxalate salts that are continuously taken in small amounts, are absorbed and enter the circulation. This oxalate combines with the calcium in body fluids and reduces the intensity of the plasma calcium as well as the red blood cells. Kidney stones formed as a result of settling of shaped crystals of calcium oxalate on to the kidney tubules, resulting in renal failure (Sharma et al., 1991; Rahman et al., 2011; Aslani et al., 2011; Rahman et al., 2013).

In the rumen, oxalates bonds with calcium, which is supplied by the other feed in the rumen, and block calcium absorption, and thus, its benefit depending on the hardness of the crystals (McKenzie et al., 1988; Rahman et al., 2011). Calcium oxalate crystal deposition in the central nervous system cause disorders like paralysis. Oxalate ions also impair the effectiveness of two essential enzymes of energy metabolism such as succinic and lactic dehydrogenase enzymes (Kaya and Pirinçci, 1995; Svedruzic, 2005). Although SBP has an important place in ruminant feed, oxalate, may be a reason for restricting its use. James et al. (1968; 1972), reported that an increase in the amount of soluble oxalate in the diet decreased the serum Ca level significantly (Aslani et al., 2011). The addition of SBP to the ration at a $20 \%$ rate, instead of barley straw, caused the serum Ca level to decrease to below the physiological limits (Balıkçı and Gürdoğan, 2002).

The low dry matter (DM) level of $10-12 \%$ of SBP prevents long-term storage. In addition, the high amount of oxalate in its content limits its consumption by animals in a short 
time and in sufficient quantities. For this reason, increasing the amount of DM by adding barley straw to SBP will facilitate the making of silage, while adding molasses will increase its aroma and facilitate its consumption. In addition, urea added in low amounts will increase the nitrogen content of the silage, thus the crude protein (CP) amount, and will prevent the $\mathrm{pH}$ from falling rapidly during fermentation. In the first days of fermentation, a suitable environment will be prepared for the binding of calcium and insoluble oxalate (Miyazaki et al, 2003) which is one of the mineral substances in the silage, at high temperatures up to $80{ }^{\circ} \mathrm{C}$ and at a $\mathrm{pH}$ that does not shift to acid. Thus, the harmful effects of oxalate that may occur after animals consume SBP will be prevented.

The purpose of the two trials in this study was to determine the effect of ensiling and adding $\mathrm{Ca}$ to SBP's soluble oxalate and determine the effects of these silages on sheep.

\section{Material and Method}

\section{Experiment I}

SBP and molasses used in the experiment were obtained from Erciş Sugar Factory, barley straw, urea and DCP were obtained from the market. By making DM analyzes of these substances, silages were prepared with DM of 20\%, 25, 30 and 35 .

One liter laboratory SBP silages (SBPS) were prepared as SBP alone and added straw at the level of DM 20, 25, 30, 35\% with the addition of 5\% molasses, $1 \%$ urea according to wet weight of SBP. Ca was addet for all of DM levels at the rate of $0,15,30,45,75$, and 105 $\mathrm{mg} / \mathrm{kg}$ wet SBP, as 5 replicates. Totally 125 silages were preperated.

Before ensiling and after 120 days of maturation, the physical properties for fleig score (Alçiçek and Özkan, 1997), Weende analysis (DM, CP, EE, ash) (Bulgurlu and Ergül, 1978), volatile fatty acids (VFA) (Hard and Horn, 1987), total oxalate, insoluble and soluble oxalate analysis (Brogren and Savage, 2003) were performed for the silages, and Fleig scores were determined (Kılıç, 1986).

This experiment was carried out according to completely randomized design and the data were evaluated using the SAS/STAT (2007) software program. The differences between groups at 1\% level were determined using the Duncan's Multiple Comparison test.

\section{Experiment II}

For the second trial, 15 tons of SBPS containing 30\% DM and $45 \mathrm{mg} / \mathrm{kg}$ SBP Ca was prepared in the concrete silo channel according to the results of experiment I. The animal material was 36 female lambs each 1-year-old. The animals were divided into 6 groups, one being the control and the remaining five as the treatment groups, according to the body weight (BW) average.

One group was given $100 \%$ SBPS, while the control group was given an SBPS-free diet. Other groups met 20\%, 40\%, 60\% and $80 \%$ of the DM consumed by the group consuming $100 \%$ SBPS from SBPS, while the other parts (80, 60, 40 and 20\%, respectively) were given from the feed DM consumed by the control group (\%50 lucerne $+\% 50$ consantreared feed). DM of SBPS and DM intake of animals were determined on a weekly basis. Moreover, BW controls, blood intake, rumen fluid samples of 4 animals randomly 
selected from each group were obtained in 14-day periods before feeding with rumen carheter.

Oxalate analyses of rumen fluids, blood samples and feed materials (Table 4) were conducted using high-performance liquid chromatography for total oxalate and insoluble oxalate. Soluble oxalate is determined as total oxalate- insoluble oxalate (Brogren and Savage, 2003). Blood Ca analyses were made with an autoanalyzer (Roche/Hitachi 912 Autoanalyzer, Roche Diagnostics $\mathrm{GmbH}$, Germany) and feed $\mathrm{Ca}$ analyses were performed with a spectrometer (AAS, THERMO Solar AA Series spectrometer). The $\mathrm{pH}$ measurements were conducted according to Hard and Horn (1978). Lactic, acetic, propionic and butyric acids in silage fluids were analyzed using high-performance liquid chromatography (Muck and Dickerson, 1988). Dry matter, CP, EE and ash (Bulgurlu and Ergül, 1978) cellulose analyses of silages (AOAC, 1990) and other feed materials were done.

This experiment was carried out according to completely randomized design too and PROC GLM in SAS/STAT (2007) was used for all data analysis. Mean treatment differences were determined by Duncan's multiple range tests with $1 \%$ level of statistical differences.

\section{Results}

\section{Experiment I}

Table 1. Soluble oxalate levels of SBP and silages

Tablo 1. ŞPP silajlarının çözülebilir okzalat seviyeleri

\begin{tabular}{lcl}
\hline Silages & Before ensiling & After ensiling \\
\hline SBP & $19.72 \pm 0.10 \mathrm{a}$ & $5.50 \pm 0.54 \mathrm{~b}$ \\
$20 \%$ DM & $3.23 \pm 0.57 \mathrm{a}$ & $3.54 \pm 0.20 \mathrm{a}$ \\
$25 \%$ DM & $5.40 \pm 0.45 \mathrm{a}$ & $4.30 \pm 0.39 \mathrm{a}$ \\
$30 \%$ DM & $6.08 \pm 0.15 \mathrm{a}$ & $2.04 \pm 0.28 \mathrm{~b}$ \\
$35 \%$ DM & $3.13 \pm 0.82 \mathrm{a}$ & $1.64 \pm 0.22 \mathrm{~b}$ \\
\hline
\end{tabular}

$\mathrm{a}, \mathrm{b}$ : Differences among values in the same line bearing different letters are significant $(\mathrm{P}<0.01)$

Table 2. Soluble oxalate $(\mathrm{mg} / \mathrm{kg})$ changes in the silage mixtures caused variations in the levels of DM and Ca levels before and after ensiling

Tablo 2. Farklı KM ve Ca seviyelerindeki karışımların silajlama öncesi ve sonrası çözülebilir okzalat $(\mathrm{mg} / \mathrm{kg})$ seviyeleri

\begin{tabular}{llll}
\hline & & \multicolumn{2}{c}{ Soluble oxalate $\mathbf{( m g / k g )}$} \\
\hline \% DM & Ca*, ppm & Before ensiling & After ensiling \\
\hline \multirow{4}{*}{20} & 0 & $3.23 \pm 0.57 \mathrm{a}$ & $3.55 \pm 0.20 \mathrm{a}$ \\
& 15 & $6.23 \pm 0.04 \mathrm{a}$ & $2.70 \pm 0.07 \mathrm{~b}$ \\
& 45 & $4.22 \pm 0.36 \mathrm{a}$ & $2.85 \pm 0.23 \mathrm{a}$ \\
& 75 & $5.41 \pm 0.88 \mathrm{a}$ & $3.66 \pm 0.03 \mathrm{~b}$ \\
& 105 & $4.68 \pm 0.23 \mathrm{a}$ & $3.06 \pm 0.15 \mathrm{~b}$ \\
\hline \multirow{4}{*}{25} & 0 & $5.40 \pm 0.47 \mathrm{a}$ & $4.30 \pm 0.39 \mathrm{a}$ \\
& 15 & $5.23 \pm 0.22 \mathrm{a}$ & $3.19 \pm 0.75 \mathrm{~b}$ \\
& 45 & $3.93 \pm 0.95 \mathrm{a}$ & $2.56 \pm 0.01 \mathrm{a}$ \\
& 75 & $3.82 \pm 0.29 \mathrm{a}$ & $2.94 \pm 0.37 \mathrm{a}$ \\
& 105 & $4.31 \pm 0.83 \mathrm{a}$ & $3.02 \pm 0.72 \mathrm{a}$ \\
\hline
\end{tabular}




\begin{tabular}{llll}
\hline & 0 & $6.08 \pm 0.15 \mathrm{a}$ & $2.04 \pm 0.28 \mathrm{~b}$ \\
30 & 15 & $5.46 \pm 0.05 \mathrm{a}$ & $1.30 \pm 0.17 \mathrm{~b}$ \\
& 45 & $9.60 \pm 0.93 \mathrm{a}$ & $1.20 \pm 0.41 \mathrm{~b}$ \\
& 75 & $4.89 \pm 0.34 \mathrm{a}$ & $2.31 \pm 1.05 \mathrm{~b}$ \\
& 105 & $7.28 \pm 1.54 \mathrm{a}$ & $1.53 \pm 0.02 \mathrm{~b}$ \\
\hline \% DM & & \multicolumn{2}{c}{ Soluble oxalate (mg/kg) } \\
\hline \multirow{4}{*}{35} & Ca*, ppm & Before ensiling & After ensiling \\
& 0 & $3.13 \pm 0.82 \mathrm{a}$ & $1.64 \pm 0.22 \mathrm{a}$ \\
& 15 & $2.69 \pm 0.56 \mathrm{a}$ & $1.31 \pm 0.11 \mathrm{a}$ \\
& 45 & $4.62 \pm 0.05 \mathrm{a}$ & $1.53 \pm 0.45 \mathrm{~b}$ \\
& 75 & $4.57 \pm 0.52 \mathrm{a}$ & $1.13 \pm 0.47 \mathrm{~b}$ \\
& 105 & $6.67 \pm 1.17 \mathrm{a}$ & $1.20 \pm 0.08 \mathrm{~b}$ \\
\hline
\end{tabular}

*: The amount of added Ca for $1 \mathrm{~kg}$ wet SBP

$\mathrm{a}, \mathrm{b}$ : Differences among values in the same line bearing different letters are significant $(\mathrm{P}<0.01)$

Table 3. Fleig Score, CP, LA and VFA contents of silages

Tablo 3. Silajların Fleig Puanları, HP, LA ve UYA içerikleri

\begin{tabular}{|c|c|c|c|c|c|c|c|}
\hline \multirow{2}{*}{ DM,\% } & \multirow{2}{*}{$\underset{*}{\text { Ca,ppm }}$} & \multirow{2}{*}{$\begin{array}{l}\text { Fleig } \\
\text { Score }\end{array}$} & \multirow{2}{*}{$\begin{array}{l}\mathbf{C P * *}, \\
\%\end{array}$} & \multicolumn{3}{|c|}{ VFA } & \multirow{2}{*}{ LA } \\
\hline & & & & $\overline{\mathbf{A A}}$ & PA & $\mathbf{B A}$ & \\
\hline \multirow{5}{*}{20} & 0 & $83.04 \pm 7.7$ & $22.64 \pm 0.6$ & $26.578 \pm 6.0$ & $6.558 \pm 15$ & $7.185 \pm 1.7$ & $59.68 \pm 8.3$ \\
\hline & 15 & $89.81 \pm 2.2$ & $21.32 \pm 0.01$ & $43.89 \pm 2.9$ & $10.91 \pm 0.9$ & $8.76 \pm 3.8$ & $36.45 \pm 5.2$ \\
\hline & 45 & $72.96 \pm 5.3$ & $24.83 \pm 0.4$ & $41.09 \pm 7.4$ & $21.52 \pm 9.1$ & $17.90 \pm 3.1$ & $19.49 \pm 9.1$ \\
\hline & 75 & $84.14 \pm 4.5$ & $23.25 \pm 0.3$ & $31.34 \pm 2.5$ & $8.87 \pm 1.3$ & $8.34 \pm 2.5$ & $51.45 \pm 4.7$ \\
\hline & 105 & $77.05 \pm 4.2$ & $23.49 \pm 0.03$ & $39.07 \pm 4.0$ & $8.78 \pm 0.9$ & $13.44 \pm 2.7$ & $38.71 \pm 6.8$ \\
\hline \multirow{5}{*}{25} & 0 & $73.73 \pm 8.5$ & $18.31 \pm 0.1$ & $58.05 \pm 7.8$ & $2.06 \pm 2.1$ & $4.67 \pm 1.7$ & $35.22 \pm 5.5$ \\
\hline & 15 & $72.11 \pm 6.8$ & $17.55 \pm 0.3$ & $30.97 \pm 7.4$ & $10.35 \pm 1.6$ & $13.38 \pm 3.3$ & $45.30 \pm 8.9$ \\
\hline & 45 & $71.50 \pm 7.4$ & $17.33 \pm 0.2$ & $58.18 \pm 2.4$ & $12.56 \pm 1.9$ & $18.76 \pm 3.9$ & $10.49 \pm 5.9$ \\
\hline & 75 & $73.13 \pm 8.5$ & $17.89 \pm 0.5$ & $39.76 \pm 8.2$ & $8.64 \pm 0.7$ & $15.89 \pm 2.8$ & $35.70 \pm 7.8$ \\
\hline & 105 & $66.32 \pm 4.3$ & $14.46 \pm 0.3$ & $31.38 \pm 7.2$ & $8.48 \pm 1.3$ & $16.42 \pm 3.9$ & $43.72 \pm 6.4$ \\
\hline \multirow{5}{*}{30} & 0 & $62.24 \pm 12.1$ & $12.87 \pm 0.01$ & $30.945 \pm 3.4$ & $6.79 \pm 0.7$ & $9.70 \pm 3.3$ & $52.56 \pm 3.5$ \\
\hline & 15 & $52.32 \pm 10.3$ & $10.35 \pm 0.1$ & $30.64 \pm 6.4$ & $9.16 \pm 2.1$ & $10.82 \pm 3.5$ & $49.38 \pm 10.7$ \\
\hline & 45 & $73.59 \pm 12.3$ & $10.55 \pm 0.1$ & $39.10 \pm 6.2$ & $11.89 \pm 1.4$ & $15.51 \pm 3.1$ & $33.49 \pm 7.4$ \\
\hline & 75 & $57.31 \pm 9.7$ & $11.61 \pm 0.1$ & $41.62 \pm 5.4$ & $10.66 \pm 1.4$ & $15.62 \pm 2.4$ & $32.10 \pm 8.6$ \\
\hline & 105 & $69.81 \pm 8.8$ & $10.82 \pm 0.3$ & $28.61 \pm 9.8$ & $15.27 \pm 5.2$ & $22.36 \pm 6.3$ & $33.76 \pm 8.0$ \\
\hline \multirow{5}{*}{35} & 0 & $82.344 \pm 4.2$ & $10.59 \pm 0.2$ & $24.95 \pm 8.2$ & $22.66 \pm 6.8$ & $18.29 \pm 4.6$ & $34.10 \pm 7.2$ \\
\hline & 15 & $60.92 \pm 10.7$ & $7.45 \pm 0.1$ & $49.50 \pm 5.0$ & $11.77 \pm 1.1$ & $12.40 \pm 1.7$ & $26.34 \pm 5.0$ \\
\hline & 45 & $60.842 \pm 14.7$ & $6.53 \pm 0.2$ & $20.30 \pm 10.8$ & $313.75 \pm 4.8$ & $.81 \pm 13.8$ & $33.14 \pm 12.0$ \\
\hline & 75 & $62.71 \pm 13.5$ & $11.35 \pm 0.2$ & $36.49 \pm 9.4$ & $12.57 \pm 3.6$ & $18.67 \pm 4.1$ & $32.27 \pm 10.1$ \\
\hline & 105 & $60.55 \pm 8.1$ & $12.51 \pm 0.03$ & $35.59 \pm 11.7$ & $716.82 \pm 2.6$ & $19.88 \pm 1.9$ & $27.70 \pm 9.1$ \\
\hline
\end{tabular}

*: The amount of added Ca for $1 \mathrm{~kg}$ wet SBP

**: Calculated amount in DM

Significant decrease in the amount of soluble oxalate was determined in the SBP (alone) silage and, silages with 30 and $35 \% \mathrm{DM}(\mathrm{P}<0.01)$. Adding barley straw, molasses, and urea to the silage, reduced the proportional amount of oxalate, and consequently, decreased the amount of soluble 
oxalate. Moreover, under the influence of ensiling (Table 1), this amount decreased in the silages with 30 and 35\% DM from 6.08 to 2.04 and from 3.13 to $1.64 \mathrm{ppm}$, respectively, $(\mathrm{P}<0.01)$ (Table 2). This decrease was mainly significant at silages with 30 and 35\% DM and added Ca 45, 75, and 105 ppm, $(\mathrm{P}<0.01)$.

Table 3 shows that the Fleig points of this silage were at gratifying and over gratifying levels, and the LA and AA were higher than the propionic and butyric acids. CP contents of silages with 30 and $35 \%$ DM were lower than others.

\section{Experiment II}

Table 4. Contents of oxalate and chemical composition of used in feedstuffs Tablo 3. Yemlemede kullanılan yem maddelerinin BM içerikleri

\begin{tabular}{lccccccccc}
\hline & DM,\% & $\begin{array}{c}\text { Totox } \\
\mathbf{m g} / \mathbf{k g}\end{array}$ & $\begin{array}{c}\text { solox } \\
\mathbf{m g} / \mathbf{k g}\end{array}$ & $\begin{array}{c}\text { insolox } \\
\mathbf{m g} / \mathbf{k g}\end{array}$ & $\begin{array}{c}\text { Ca, } \\
\text { \% }\end{array}$ & Ash,\% & EE,\% & CP,\% & CC,\% \\
\hline Alfalfa straw & 92.00 & 62.31 & 8.20 & 54.10 & 1.35 & 8.97 & 1.58 & 16.30 & 35.78 \\
$\begin{array}{l}\text { Concentrated } \\
\text { feed }\end{array}$ & 90.49 & 15.98 & 6.13 & 9.849 & 2.45 & 13.86 & 2.35 & 11.90 & 8.12 \\
SBPS & 92.83 & 30.93 & 7.29 & 23.642 & 0.73 & 10.87 & 0.98 & 13.90 & 33.24 \\
\hline
\end{tabular}

Totox: Total oxalate, solox: soluble oxalate, insolox: insoluble oxalate

Table 5. Nutrients intake of groups according to time

Tablo 5. Gruplarin zamana göre besin madde tüketimleri

\begin{tabular}{|c|c|c|c|c|c|c|c|c|}
\hline Groups & Day & $\begin{array}{l}\text { Totox } \\
(\mathrm{mg} / \mathrm{kg})\end{array}$ & $\begin{array}{l}\text { solox } \\
(\mathrm{mg} / \mathrm{kg})\end{array}$ & $\mathrm{Ca}(\mathrm{g})$ & CP (g) & $\mathrm{CC}(\mathrm{g})$ & EE (g) & $\operatorname{ash}(\mathrm{g})$ \\
\hline \multirow{4}{*}{ Control } & 14 & $559 \pm 11,1 \mathrm{ab}$ & $102 \pm 2,0 \mathrm{ab}$ & $271 \pm 5,4 b$ & $2013 \pm 40,2 \mathrm{ab}$ & $3135 \pm 62,6 \mathrm{a}$ & $281 \pm 5,6 b$ & $630 \pm 32 \mathrm{ab}$ \\
\hline & 28 & $664 \pm 13,2 \mathrm{a}$ & $117 \pm 2,3 \mathrm{a}$ & $313 \pm 6,2 \mathrm{a}$ & $2321 \pm 46,3 \mathrm{a}$ & $3614 \pm 72,1 \mathrm{a}$ & $324 \pm 5,3 \mathrm{a}$ & $1879 \pm 37 \mathrm{a}$ \\
\hline & 42 & $532 \pm 10,6 b$ & $97 \pm 1,9 b$ & $259 \pm 5,1 b$ & $1918 \pm 38,3 b$ & $2986 \pm 59,6 \mathrm{a}$ & $268 \pm 5,3 b$ & $1553 \pm 31 b$ \\
\hline & $\bar{X}$ & $579 \pm 6,6 \mathrm{~A}$ & $106 \pm 1.2 \mathrm{~A}$ & $281 \pm 3.4 \mathrm{~A}$ & $2084 \pm 24.0$ & $3245 \pm 37.4 \mathrm{D}$ & $291 \pm 3.3 \mathrm{~A}$ & $1687 \pm 19 A$ \\
\hline \multirow{4}{*}{$20 \%$} & 14 & $534 \pm 10,6 \mathrm{ab}$ & $102 \pm 2,0 \mathrm{ab}$ & $238 \pm 4,7 \mathrm{ab}$ & $2004 \pm 40,0 \mathrm{ab}$ & $3449 \pm 68,9 \mathrm{ab}$ & $252 \pm 5,0 \mathrm{ab}$ & $1611 \pm 32 \mathrm{ab}$ \\
\hline & 28 & $619 \pm 12,3 \mathrm{a}$ & $119 \pm 2,3 \mathrm{a}$ & $276 \pm 5,5 \mathrm{a}$ & $2323 \pm 46,3 a$ & $3998 \pm 79,8 \mathrm{a}$ & $293 \pm 5,8 \mathrm{a}$ & $1868 \pm 37 \mathrm{a}$ \\
\hline & 42 & $510 \pm 10,1 b$ & $98 \pm 1,9 b$ & $208 \pm 4,1 b$ & $1914 \pm 38,2 b$ & $3295 \pm 65,8 b$ & $241 \pm 4,8 b$ & $1538 \pm 30 b$ \\
\hline & $\bar{X}$ & $555 \pm 6.3 \mathrm{AB}$ & $106 \pm 1.2 \mathrm{~A}$ & $240 \pm 2.7 \mathrm{ABC}$ & $2080 \pm 23.9$ & $3581 \pm 41.2 \mathrm{CD}$ & $262 \pm 3.0 \mathrm{~A}$ & $1672 \pm 19 \mathrm{~A}$ \\
\hline \multirow{4}{*}{$40 \%$} & 14 & $511 \pm 10,2 \mathrm{ab}$ & $103 \pm 2,0 \mathrm{a}$ & $205 \pm 4,0 \mathrm{a}$ & $2000 \pm 39,9 \mathrm{ab}$ & $3774 \pm 75,3 \mathrm{ab}$ & $224 \pm 4,4 \mathrm{al}$ & $1597 \pm 31 \mathrm{ab}$ \\
\hline & 28 & $591 \pm 11,8 \mathrm{a}$ & $118 \pm 2,3 \mathrm{a}$ & $235 \pm 4,6 a$ & $2303 \pm 45,9 a$ & $4362 \pm 87,1 \mathrm{a}$ & $257 \pm 5,1 \mathrm{a}$ & $1854 \pm 37 \mathrm{a}$ \\
\hline & 42 & $487 \pm 9,7 b$ & $98 \pm 1,9 b$ & $195 \pm 3,9 a$ & $1906 \pm 38,0 \mathrm{~b}$ & $3598 \pm 71,8 b$ & $214 \pm 4,2 b$ & $1522 \pm 30 b$ \\
\hline & $\bar{X}$ & $530 \pm 6.1 \mathrm{AB}$ & $106 \pm 1.2 \mathrm{~A}$ & $212 \pm 2.4 \mathrm{BC}$ & $2070 \pm 23.8$ & $3911 \pm 45.1 \mathrm{BC}$ & $232 \pm 2.6 \mathrm{C}$ & $1651 \pm 19 A$ \\
\hline \multirow{4}{*}{$60 \%$} & 14 & $488 \pm 9,7 \mathrm{ab}$ & $103 \pm 2,0 \mathrm{a}$ & $172 \pm 3,4 b$ & $1994 \pm 39,8 \mathrm{ab}$ & $4096 \pm 81,8 \mathrm{ab}$ & $196 \pm 3,9 \mathrm{al}$ & $1581 \pm 31 \mathrm{ab}$ \\
\hline & 28 & $547 \pm 10,9 \mathrm{a}$ & $110 \pm 2,2 \mathrm{a}$ & $441 \pm 8,8 \mathrm{a}$ & $2303 \pm 46,0 \mathrm{a}$ & $4700 \pm 93,8 \mathrm{a}$ & $227 \pm 4,5 \mathrm{a}$ & $1823 \pm 36 \mathrm{a}$ \\
\hline & 42 & $453 \pm 9,0 \mathrm{~b}$ & $97 \pm 1,9 . \mathrm{a}$ & $161 \pm 3,2 b$ & $1870 \pm 37,2 b$ & $3838 \pm 76,6 b$ & $184 \pm 3,6 b$ & $1488 \pm 29 b$ \\
\hline & $\bar{X}$ & $496 \pm 5.7 \mathrm{~B}$ & $103 \pm 1.1 \mathrm{~A}$ & $258 \pm 2.9 \mathrm{AB}$ & $2055 \pm 23.7$ & $4211 \pm 48.5 \mathrm{AB}$ & $202 \pm 2.3 \mathrm{D}$ & $1631 \pm 18 \mathrm{~A}$ \\
\hline \multirow{4}{*}{$80 \%$} & 14 & $452 \pm 9,02 \mathrm{a}$ & $99 \pm 1,9 a$ & $138 \pm 2,7 b$ & $1985 \pm 39,6 \mathrm{ab}$ & $4397 \pm 87,8 \mathrm{ab}$ & $168 \pm 3,3 \mathrm{a}$ & $1560 \pm 31 \mathrm{ab}$ \\
\hline & 28 & $409 \pm 8,1 \mathrm{ab}$ & $76 \pm 1,5 b$ & $332 \pm 6,6 a$ & $2262 \pm 45,1 \mathrm{a}$ & $4894 \pm 97,7 \mathrm{a}$ & $191 \pm 3,8 \mathrm{a}$ & $1751 \pm 34 \mathrm{a}$ \\
\hline & 42 & $339 \pm 6,7 b$ & $64 \pm 1,2 b$ & $129 \pm 2,5 b$ & $1863 \pm 37,2 b$ & $4039 \pm 80,6 b$ & $157 \pm 3,1 \mathrm{a}$ & $1443 \pm 28 b$ \\
\hline & $\bar{X}$ & $400 \pm 5.6 \mathrm{C}$ & $79 \pm 0.9 \mathrm{~B}$ & $200 \pm 2.3 \mathrm{C}$ & $2037 \pm 23.4$ & $4443 \pm 51.2 \mathrm{~A}$ & $172 \pm 1.9 \mathrm{E}$ & $1584 \pm 18$ \\
\hline \multirow{4}{*}{$100 \%$} & 14 & $389 \pm 7,7 \mathrm{a}$ & $86 \pm 1,7 \mathrm{a}$ & $102 \pm 2,0 \mathrm{~b}$ & $1967 \pm 39,2 \mathrm{ab}$ & $5071 \pm 101,2 \mathrm{a}$ & $138 \pm 2,7 \mathrm{a}$ & $1519 \pm 30 \mathrm{ab}$ \\
\hline & 28 & $254 \pm 7,7 b$ & $32 \pm 0,6 \mathrm{~b}$ & $222 \pm 4,4 \mathrm{a}$ & $2216 \pm 44,2 \mathrm{a}$ & $4657 \pm 93,0 \mathrm{a}$ & $154 \pm 3,0 \mathrm{a}$ & $1638 \pm 32 \mathrm{a}$ \\
\hline & 42 & $37 \pm 0,7 \mathrm{c}$ & $-34 \pm 0,6 c$ & $78 \pm 1,5 b$ & $1776 \pm 35,4 b$ & $3905 \pm 78,0 \mathrm{~b}$ & $121 \pm 2,4 \mathrm{a}$ & $1247 \pm 24 b$ \\
\hline & $\bar{X}$ & $227 \pm 2.6 \mathrm{D}$ & $28 \pm 0.3 \mathrm{C}$ & $134 \pm 1.5 \mathrm{D}$ & $1987 \pm 22.9$ & $4544 \pm 52.4 \mathrm{~A}$ & $138 \pm 1.5 \mathrm{~F}$ & $1468 \pm 16 \mathrm{~B}$ \\
\hline
\end{tabular}

$\mathrm{A}, \mathrm{B}, \mathrm{C} ; \mathrm{a}, \mathrm{b}, \mathrm{c}$ : Differences among values in the same column bearing different letters are significant $(\mathrm{P}<0.01)$

Totox: Total oxalate, solox: soluble oxalate 
Table 6. Blood parameters and body weight changes

Tablo 6. Kan parametreleri ve canlı ağırlık değişimleri

\begin{tabular}{|c|c|c|c|c|}
\hline \multirow[b]{2}{*}{ Groups } & \multirow[b]{2}{*}{ Day } & \multicolumn{2}{|c|}{ Blood } & \multirow[b]{2}{*}{ BW, kg } \\
\hline & & Totox, mg/dl & Ca, mg/dl & \\
\hline \multirow{5}{*}{ Control } & 0 & $1.57 \pm 0,05 \mathrm{a}$ & $8.35 \pm 0,31 \mathrm{a}$ & $26.26 \pm 0,98 b$ \\
\hline & 14. & $1.65 \pm 0,06 \mathrm{a}$ & $7.85 \pm 0,29 \mathrm{a}$ & $29.17 \pm 1,09 \mathrm{ab}$ \\
\hline & 28. & $1.54 \pm 0,05 \mathrm{a}$ & $8.13 \pm 0,30 \mathrm{a}$ & $31.07 \pm 1,16 \mathrm{ab}$ \\
\hline & 42. & $1.57 \pm 0,05 \mathrm{a}$ & $7.50 \pm 0,28 \mathrm{a}$ & $31.30 \pm 1,16 \mathrm{a}$ \\
\hline & $\bar{X}$ & $1.58 \pm 0,02 \mathrm{~A}$ & $7.96 \pm 0,14 \mathrm{~B}$ & $29.45 \pm 0,55$ \\
\hline \multirow{5}{*}{$20 \%$} & 0. & $1.37 \pm 0,05 \mathrm{a}$ & $8.38 \pm 0,31 \mathrm{a}$ & $26.21 \pm 0,97 b$ \\
\hline & 14. & $1.28 \pm 0,04 \mathrm{a}$ & $8.28 \pm 0,30 \mathrm{a}$ & $28.53 \pm 1,06 \mathrm{ab}$ \\
\hline & 28. & $1.30 \pm 0,05 \mathrm{a}$ & $7.70 \pm 0,30 \mathrm{a}$ & $30.87 \pm 1,22 \mathrm{ab}$ \\
\hline & 42. & $1.16 \pm 0,04 \mathrm{a}$ & $7.83 \pm 0,29 \mathrm{a}$ & $31.30 \pm 1,16 \mathrm{a}$ \\
\hline & $\bar{X}$ & $1.27 \pm 0,02 \mathrm{~B}$ & $8.05 \pm 0,15 \mathrm{~B}$ & $29.23 \pm 0,54$ \\
\hline \multirow{5}{*}{$40 \%$} & 0. & $1.53 \pm 0,05 \mathrm{a}$ & $8.38 \pm 0,31 \mathrm{a}$ & $25.83 \pm 0,96 b$ \\
\hline & 14. & $1.61 \pm 0,06 \mathrm{a}$ & $8.30 \pm 0,31 \mathrm{a}$ & $28.20 \pm 1,05 \mathrm{ab}$ \\
\hline & 28. & $1.36 \pm 0,05 \mathrm{a}$ & $7.97 \pm 0,29 \mathrm{a}$ & $30.90 \pm 1,15 \mathrm{ab}$ \\
\hline & 42. & $1.34 \pm 0,05 \mathrm{a}$ & $8.00 \pm 0,29 \mathrm{a}$ & $31.53 \pm 1,17 \mathrm{a}$ \\
\hline & $\bar{X}$ & $1.47 \pm 0,02 \mathrm{~A}$ & $8.16 \pm 0,15 \mathrm{AB}$ & $29.12 \pm 0,54$ \\
\hline \multirow{5}{*}{$60 \%$} & 0. & $1.55 \pm 0,05 \mathrm{a}$ & $8.48 \pm 0,31 \mathrm{a}$ & $26.03 \pm 0,97 b$ \\
\hline & 14. & $1.47 \pm 0,05 \mathrm{a}$ & $8.40 \pm 0,31 \mathrm{a}$ & $28.87 \pm 1,07 \mathrm{ab}$ \\
\hline & 28. & $1.46 \pm 0,05 \mathrm{a}$ & $8.10 \pm 0,30 \mathrm{a}$ & $30.87 \pm 1,15 \mathrm{ab}$ \\
\hline & 42. & $1.46 \pm 0,05 \mathrm{a}$ & $8.12 \pm 0,30 \mathrm{a}$ & $31.87 \pm 1,19 \mathrm{a}$ \\
\hline & $\overline{\mathrm{X}}$ & $1.49 \pm 0,02 \mathrm{~A}$ & $8.28 \pm 0,15 \mathrm{AB}$ & $29.41 \pm 0,54$ \\
\hline \multirow{5}{*}{$80 \%$} & 0. & $1.65 \pm 0,06 \mathrm{a}$ & $8.43 \pm 0,31 \mathrm{a}$ & $26.36 \pm 0,98 b$ \\
\hline & 14. & $1.52 \pm 0,05 \mathrm{a}$ & $8.55 \pm 0,31 \mathrm{a}$ & $29.03 \pm 1,08 \mathrm{ab}$ \\
\hline & 28. & $1.55 \pm 0,05 \mathrm{a}$ & $7.95 \pm 0,29 \mathrm{a}$ & $31.30 \pm 1,16 \mathrm{ab}$ \\
\hline & 42. & $1.59 \pm 0,05 \mathrm{a}$ & $7.86 \pm 0,29 a$ & $31.80 \pm 1,18 \mathrm{a}$ \\
\hline & $\bar{X}$ & $1.58 \pm 0,02 \mathrm{~A}$ & $8.21 \pm 0,15 \mathrm{AB}$ & $29.62 \pm 0,553$ \\
\hline \multirow{5}{*}{$100 \%$} & 0. & $1.79 \pm 0,06 \mathrm{a}$ & $8.50 \pm 0,31 \mathrm{ab}$ & $25.99 \pm 0,97 b$ \\
\hline & 14. & $1.35 \pm 0,05 b$ & $9.12 \pm 0,34 \mathrm{a}$ & $27.67 \pm 1,03 b$ \\
\hline & 28. & $1.22 \pm 0,04 \mathrm{bc}$ & $8.35 \pm 0,31 \mathrm{ab}$ & $29.03 \pm 1,08 b$ \\
\hline & 42. & $1.51 \pm 0,05 \mathrm{ab}$ & $8.02 \pm 0,29 \mathrm{~b}$ & $28.93 \pm b$ \\
\hline & $\overline{\mathrm{X}}$ & $1.47 \pm 0,02 \mathrm{~A}$ & $8.50 \pm 0,15 \mathrm{~A}$ & $27.91 \pm 0,52$ \\
\hline
\end{tabular}

A, B, C; a, b, c: Differences among values in the same column bearing different letters are significant $(\mathrm{P}<0.01)$ BWC: Body weight change; Totox: Total oxalate Ca: Calcium

The nutrient contents of feed raw materials used in animal feeding are as in Table 4. Table 5 shows time of increase in the consumption of SBPS in the ration, and the decrease in the intake of total oxalate, soluble oxalate, $\mathrm{Ca}, \mathrm{CP}, \mathrm{EE}$, and crude ash, on average $(\mathrm{P}<0.01)$. Conversely, the amount of cellulose intake increased significantly $(\mathrm{P}<0.01)$ because the amount of cellulose was increased by adding barley straw into the silages. Since the rations contents of $\mathrm{CP}$ were close to each other, $\mathrm{CP}$ intake did not decrease significantly $(\mathrm{P}>0.01)$. Ash intake was decreased by only consuming $100 \%$ of SBPS group $(\mathrm{P}<0.01)$. 
Nutrient intake, determined according to time, particularly after the $28^{\text {th }}$ day, decreased except for $\mathrm{EE}$ for 80 and $100 \%$, Ca for $40 \%$ SBPS consumed groups $(\mathrm{P}<0.01)$. The reason for this is the decrease in DM intake of $100 \%$ SBPS group from time to time, whereas the other group's DM consumption was limited compared to this group. Specifically, after day 28, in 100 and $80 \%$ groups, the selectivity was increased while the DM intake decreased. The animals selected the SBP tracks but not barley straw from the silage. The calculation of soluble oxalate intake of the $100 \%$ group was relatively negative because the total oxalate of the silage was concentrated in the barley hay particles. The soluble oxalate was not determined in blood and rumen fluid samples.

In Table 6, differences in the blood total oxalate values among the groups were insignificant except for $20 \%$ group $(\mathrm{P}<0.01)$. The highest blood calcium level was in the $100 \%$ group, the lowest levels were in the control and $20 \%$ groups, the differences between the other groups were insignificant $(\mathrm{P}<0.01)$. It can be regarded as acceptable because the extra-added $\mathrm{Ca}$ in the silage increased the utilization of $\mathrm{Ca}$ from the ration's bonding of the soluble oxalate of SBP and increased the blood Ca level.

Body weight changes were examined, and the differences among the groups were found to be insignificant $(\mathrm{P}<0.01)$. Depending on the time, the differences between the total blood oxalate and $\mathrm{Ca}$ were not significant except in the $100 \%$ group. The change in body weight change, according to time, was not significant in the $100 \%$ group, but the reduction in body weight increases, particularly after the $28^{\text {th }}$ day $(\mathrm{P}<0.01)$.

\section{Discussion and Conclusion}

Since the silage material does not have strength, the air was completely removed. High sugar content has accelerated fermentation. Due to the alkaline structure of the SBP, straw, and urea, the pH value was higher in the early days. This can be attributed to the increase in the binding ability of soluble oxalate with $\mathrm{Ca}, \mathrm{Mg}$, and $\mathrm{Fe}$ minerals at high $\mathrm{pH}$ and temperature in the first days of silages (Gündüz, 1993). Thus, the amount of soluble oxalate was decreased (Table 2) and the insoluble oxalate was increased.

Regarding the quality of silage in the investigation, (Table 3) it was shown that this silages might be used safely in feeding since Fleig points were persuasive and over gratifying level, and LA and AA in total VFA were higher than propionic and butyric acids. Although low CP contents of 30 and 35\% DM silages were caused by the proportional amount of additional urea according to the SBP amount in silages, the CP levels of these silages were at appropriate levels.

In experiment II, the soluble oxalate was not determined in the blood and rumen fluid samples. This circumstance showed that soluble oxalate amount of SBP was decreased to a tolerable level by the rumen oxalate degrading microorganisms. Since there is no direct link between the total oxalate amount of blood and consumed oxalate, the total blood oxalate levels of all groups were similar. This situation was reported by James et al. (1972) as blood total oxalate level is affected by not only feed oxalate amount but also by oxalate produced by metabolism, which maintains a constant total oxalate level. There were no differences in the $\mathrm{Ca}$ and total oxalate average values among the control and other maintenance groups suggesting that adding SBPS to rations did not affect the $\mathrm{Ca}$ and oxalate metabolisms. Thus, it was determined that adding Ca to SBPS can eliminate the toxic effect of the soluble oxalate in SBP. In a similar study, Rahman et al. (2011) reported that when the oxalate-rich napiergeass forage plant was grown with Ca fertilizer, the amount of soluble oxalate and total oxalate in the plant decreased, and the blood calcium levels increased without affecting the feed consumption, blood magnesium level and body weight gain of the animals consuming this feed. As a result of the study, they concluded that the negative effects of this plant on animals caused by oxalate can be minimized by $\mathrm{Ca}$ fertilization. 
These results indicate that ensiling the sugar beet pulp for increasing DM and adding Ca decreased the soluble oxalate to a level that can be tolerated by ruminants. Thus, the toxic effects are eliminated and added to sheep ratio as $60 \%$. Conversely, the consumption of this silage alone can be undesirable because of selectivity and anorexic issues in animals.

\section{Acknowledgements}

This work is supported by the Scientific Research Projects Coordination Unit of Yüzüncü Y11 University under the Grant [376-206-ZF-B01].

\section{References}

AOAC, 1990. Association of official analytical chemistry. Official Methods of Analysis, $15^{\text {th, }}$ Vol:1, Washington,D.C.

Alçiçek, A. and Özkan, K. 1997. Determination of silage quality with physical and chemical methods in silages. Turkey I. Silage Congress, 16-19 September Bursa. 241-246.

Aslani, M.R., Movassaghi, A.R., Najarnezhad, V., Pirouz, H.J. and Bami, M.H. 2011. Acute oxalate intoxication associated to ingestion of eshnan (Seidlitzia rosmarinus) in sheep. Trop Anim Health Prod:43:1065-1068 DOI 10.1007/s11250-011-9818-0.

Azcarate-Peril, M.A.; Bruno-Ba'rcena, J.M.; Hassan, H.M. and Klaenhammer, T.R. 2006. Transcriptional and functional analysis of oxalyl-coenzyme A (CoA) decarboxylase and formyl-CoA transferase genes from Lactobacillus acidophilus. Applied and Environmental Microbiology. 1891-1899.

Balikci, E. and Gürdogan, F. 2002. The effect of wet sugar beet pulp fed as the only forage source on some heamatologic and biochemicial parameters in lambs. YYU Veterinary Faculty Journal, 13 (1-2):5053.

Brogren, M. and Savage, G.P. 2003. Bioavailability of soluble oxalate from spinach eaten with and without milk products. Asia Pacific Journal of Clinical Nutrition, 12 (2): 219-224.

Bulgurlu, S. and Ergul, M. 1978. Analyses methods of physical, chemical and biological of feeds. Ege University Press, Issue Number: 127,176p

Gündüz, T. 1993. The Quantitative Analysis Laboratory Book. Bilge Publications, Ankara.

Hart, S.P. and Horn, F.P. 1987. Ensiling characteristics and digestibility of combinations of turnips and wheat straw. Journal of Animal Science, 64:1790-1800.

James, L.F., Street, J.C.and Butcher, J.E. 1972. Halogeton poisoning of sheep: Effect of high level oxalate intake. Journal of Animal Science, 35 (6): 1233-8

James, L.F., Street, J.C., Butcher, J.E. and Shupe, J.L. 1968. Intake on electrolyte metabolism Glomeratus halogeton. Oxalate Metabolism in Sheep II. Effect of Low Level. Journal of Animal Science, 27:724-729.

Kaya, S. and Pirincci, L. 1995. Veterinary Clinical Toxicology. Glycosides. Medisan Publications, Ankara.

Kilic, A. 1986. Silage . Bilgehan Printing House. İzmir, Turkey 350 p.

Mc Kenzie, R.A., Bell, A.M., Storie, G.J., Kenan, F.J., Cornack, K.M. and Grant, S.G. 1988. Acute oxalate poisoning of sheep by buffelgrass (Cenchrus ciliaris). Australian Veterinary Jornal, 65: 26.

Miyazaki, S., Yamanake, N. and Guruge,.K. 2003. Simple capillary electrophoretic determination of soluble oxalate and nitrate in forages grasses. Journal of Veterinary Diagnostic Investigation, $15: 480-483$ 
Muck, R.E. and Dickerson, J.T. 1988. Storage temperature effects on proteolysis in alfalfa silage. Transactions of the American Society of Agriculture Engineering, 31:1005-1009.

Rahman M.M., Abdullah, R.B. and Wan Khadijah, W.E. 2013. A review of oxalate poisoning in domestic animals: tolerance and performance aspects. Journal of Animal Physiology and Animal Nutrition, 97:605-614.

Rahman, M. M., Nakagawa, T., Niimi, M., Fukuyama, K. and Kawamura, O. 2011. Effects of Feeding Oxalate Containing Grass on Intake and the Concentrations of Some Minerals and Parathyroid Hormone in Blood of Sheep. Asian-Aust. J. Anim. Sci. Vol. 24, No. 7 : 940 - 945

S.A.S., 2007. SAS® User's Guide: Statistics, Version 8. SAS Institute Inc., Cary, NC.

Sharma, S., Vaidyanathan, S., Nath, R. and Thind, S.K. 1991. Advances in pathophysiology of calcium oxalate stone disease. Indian Journal of Urology, 8: 25-37.

Svedruzic, D., Jo'nssona, S., Toyota, C.G., Reinhardtb, L.A., Ricagnoc, S., Lindqvistb, Y. and Richards, N.G.J. 2005. The enzymes of oxalate metabolism: unexpected structures and mechanisms. Archives of Biochemistry and Biophysics. 433:176-192. 